\title{
Pesticides et métaux lourds dans l'eau de boisson, les sols et les sédiments de la ceinture cotonnière de Gogounou, Kandi et Banikoara (Bénin)
}

\author{
Soulé ADAM ${ }^{1}$, Patrick A. EDORH ${ }^{2 *}$, Henri TOTIN ${ }^{3}$, Luc KOUMOLOU ${ }^{4}$, Ernest \\ AMOUSSOU $^{3}$, Kodjo AKLIKOKOU ${ }^{5}$ et Michel BOKO ${ }^{3}$ \\ ${ }^{I}$ Ministère de l'Agriculture, de l'Elevage et de la Pêche (MAEP), Bénin. \\ ${ }^{2}$ Département de Biochimie et de Biologie Cellulaire, Université d'Abomey-Calavi (UAC), 01 BP 526 Cotonou, \\ Bénin. \\ ${ }^{3}$ Laboratoire Pierre Pagney : Climat, Eau, Ecosystème et Développement (LACEEDE), 03 BP 1122 Cotonou, \\ Bénin. \\ ${ }^{4}$ Département de Physiologie/Pharmacologie, Faculté des Sciences, Université de Lomé (UL), BP 1515 Lomé, \\ Togo. \\ ${ }^{5}$ Département de Physiologie/Pharmacologie, Centre de Recherche et de Formation sur les Plantes \\ Médicinales (CERFOPLAM), Faculté des Sciences, Université de Lomé (UL), BP 1515 Lomé, Togo. \\ *Auteur correspondant,; E-mail : patrickedorh@yahoo.fr; Tél. (00229)97071596
}

\section{RESUME}

Dans cette expérimentation, nous avons évalué la contamination de l'eau de boisson, du sol et des sédiments par les métaux lourds et les pesticides liés à la culture du coton dans la zone cotonnière de Gogounou, Kandi et Banikoara (Bénin). Les résidus de pesticides (pyréthrinoïdes, insecticides et organophosphorés) sont recherchés par chromatographie en phase gazeuse ; les métaux lourds (cadmium, plomb, arsenic, nickel, zinc et mercure), par spectrophotométrie d'absorption atomique. Les résultats ont montré dans les trois régions une contamination régulière de l'eau de pompe par les métaux à l'exception du mercure $(0.000 \mathrm{ppb})$ et de l'arsenic $(0.000 \mathrm{ppb})$. Les teneurs les plus élevées avoisinent $0,126 \mathrm{ppb}$ et $0,100 \mathrm{ppb}$ respectivement pour le plomb et le zinc. Pour les pesticides, la contamination est aussi régulière pour chaque matière active au sein d'une même famille de pesticides. Les teneurs en cyfluthrine des pyréthrinoïdes tournent autour de 0,200 ppm dans les sédiments et celles de l'endosulfan des organophosphorés, autour de 0,080 ppm dans tous les sols. Mais les concentrations de métaux lourds et de pesticides sont plus élevées dans les sédiments que dans les sols.

(C) 2010 International Formulae Group. All rights reserved.

Mots clés : Culture cotonnière, sol, eau de pompe, sédiments, métaux lourds, pesticides.

\section{INTRODUCTION}

La croissance démographique a entraîné une réduction des aires de culture dans la ceinture cotonnière de Gogounou, Kandi et Banikoara, dans le Nord du Bénin (Aboudou et Gibiyaye, 2000). En effet, depuis
1990, le nombre d'exploitants agricoles dans la ceinture cotonnière n'a cessé d'augmenter (Adam, 2005). Et une augmentation de la population agricole sur une terre cultivable réduite conduit à sa surexploitation. 
Par ailleurs, la filière coton constitue la base de l'économie rurale et agro-industrielle au Bénin. Sa contribution, en terme de valeur ajoutée, est estimée à $13 \%$ du PIB. Elle représente entre $70 \%$ et $80 \%$ de la valeur totale des exportations et $35 \%$ des rentrées fiscales (CIPPB, 2008 ; Gbesso, 2000 ; Ton et Vodouhè, 1994). Mais la pression parasitaire induit une perte de récolte de l'ordre de $20 \%$ (Ferron et al., 2006). Face à ces enjeux économiques, tous les moyens, tel que l'utilisation des pesticides, sont justifiés par les agriculteurs, pourvu qu'ils permettent d'augmenter le rendement du coton. Encore que le nombre d'exploitants n'est pas proportionnel au nombre d'encadreurs sur le terrain. En effet, $42 \%$ des exploitants agricoles ne bénéficient pas d'encadrement technique pour l'usage des engrais et des pesticides dans les communes de Gogounou, Kandi et Banikoara (Adam, 2005). Et pour accentuer indirectement le phénomène, l'Etat a libéralisé depuis 1992 la sous-filière intrants par l'agrément d'importateurs et de distributeurs privés (CIPPB, 2008). Actuellement, certaines molécules classées $1 \mathrm{~b}$ (hautement dangereux) ou $\mathrm{T}$ (toxiques) comme l'endosulfan ou le triazophos sont couramment utilisées avec des risques pour la faune du sol, la qualité des cultures vivrières adjacentes ( Biaou et al., 2003) et des eaux de surface (DIREN, 2003). Les conséquences immédiates sont une atteinte à la santé humaine (Jager-Mischke, 1993 ; Toe, 2004) et une dégradation de l'environnement (Van der Werf, 1997). Les atteintes sanitaires se traduisent par des maladies diverses causées par le contact avec des pesticides et par la consommation de produits alimentaires, poissons, viandes, légumes, fruits contaminés (Jager-Mischke, 1993 ; Cisse, 2003 ; Nebie, 2002 ; Soclo, 2005 ; Tapsoba et BonziCoulibaly, 2006).

Au Bénin, Biaou et al. (2003) et Pazou et al. (2006) ont étudié la contamination des composantes environnementales par les pesticides utilisés dans les milieux agricoles. Seulement, ces auteurs n'ont pas pris en compte la contamination des eaux de surface ou souterraine, ni par les pesticides, ni par les métaux lourds. Or, la contamination des eaux de surface ou souterraine par les toxiques est étroitement liée a celle du sol (Coats, 1991); et les pesticides, en l'occurrence les fongicides, contiennent des métaux lourds (Deluisa et al., 1996 ; Bourrelier et Berthelin, 1998) qui peuvent s'ajouter aux contaminants déjà existants dans le sol.

L'objectif de notre étude est donc de rapprocher la contamination en pesticides et en métaux lourds des sols agricoles à la contamination de l'eau de boisson (eau souterraine) et de celle des eaux de surface (à travers l'analyse des sédiments). L'étude a ainsi permis d'évaluer l'impact des traitements agricoles sur l'environnement voire la santé humaine et animale.

\section{MATERIEL ET METHODES Cadre d'étude}

La ceinture cotonnière BanikoaraKandi-Gogounou (Figure 1) est située entièrement dans le Département de l'Alibori et plus précisément entre $9^{\circ} 30^{\prime}$ et $12^{\circ} 30^{\prime}$ Latitude Nord et entre $0^{\circ} 30^{\prime}$ et $3^{\circ} 45^{\prime}$ Longitude Est (Adam, 2005).

La ceinture cotonnière de GogounouKandi-Banikoara bénéficie du domaine climatique soudano-sahélien, avec un régime unimodal caractérisé par deux saisons contrastées qui font de cette région, un milieu peu favorable aux exploitations agricoles. Cependant, selon Guiwa (1996), la saison sèche est plus rude à Kandi et à Banikoara $(13,34 \mathrm{~mm} / \mathrm{mois})$ qu'à Gogounou $(24,13$ $\mathrm{mm} / \mathrm{mois}$ ). Il existe une variété de sols dans la région (Adam, 2005). Or la nature du sol et la pluviosité sont connus comme des facteurs qui influencent la teneur des pesticides et métaux lourds dans le sol (Savadogo et al., 2006). Par ailleurs, les quantités et les qualités des pesticides utilisés dans les cultures varient 
d'une commune à l'autre dans la ceinture cotonnière de Gogounou-Kandi-Banikoara (Adam, 2005).

Les sols de la ceinture cotonnière Banikoara- Kandi- Gogounou, tout comme ceux de la partie septentrionale du pays, résultent directement du processus naturel de ferrallitisation et de ferrugination. Ces processus sont complétés par trois types de maniements à savoir: le lessivage, l'appauvrissement et l'induration qui sont le résultat de l'altération des formations rocheuses mises en place. Il s'agit des sols ferrugineux, sablo-limoneux, limoneux, concrétionnés, écroûtés et rocailleux (Adam, 2005). Ces sols, au regard des nuances qu'ils présentent, constituent les atouts à la production agricole.

\section{Echantillonnage}

Des prélèvements de sol, d'eau de robinet et de sédiments pris dans les retenues d'eau environnantes ont été réalisés pendant la saison sèche sur plusieurs sites pour être dosés en métaux lourds (cadmium, plomb, arsenic, nickel, zinc et mercure) et en résidus de pesticides (pyréthrinoïdes, insecticides, organophosphorés et organochlorés). Les échantillons d'eau de boisson, parce que directement liée à la santé, ont été réalisés au niveau du réseau d'adduction d'eau potable de chacune des trois régions. Au niveau des champs de coton a été prélevé le sol tandis que les bas-fonds ou cuvette des retenues d'eau ont fait l'objet de prélèvement de sédiment. Les sédiments sont préférés aux eaux interstitielles parce qu'ils constituent le lieu de dépôt de tout ce qui est transporté du continent. Leur qualité toxicologique témoigne donc du niveau de pollution des activités anthropiques (Gnandi et al., 2007). En effet, les eaux de surface peuvent transporter des détritus pollués et toxiques (engrais, pesticides) qui altèrent la qualité de l'eau et des sédiments (Saad, 1985).

\section{Traitement et analyse}

Le sol, durci à cause de la saison sèche, est ameubli et mis dans des flacons de $7 \mathrm{ml}$ stérilisés, étiquetés et conservés au four électrique à $50{ }^{\circ} \mathrm{C}$ pour séchage. Les sédiments ont été directement prélevés à 75 $\mathrm{cm}$ de la surface de l'eau et à $100 \mathrm{~cm}$ de la bordure. Tous les échantillons sont conservés dans un réfrigérateur à une température de 0 ${ }^{\circ} \mathrm{C}$.

La méthode utilisée pour détecter les pesticides ou leurs dérivés et leur concentration dans les échantillons prélevés est la Chromatographie/Lead suivant la méthode de Kammerbauer et Moncada (1998), Edmond et al. (1985) et Wolska et al. (1999). Les substances actives recherchées sont contenues dans la gamme variée de pesticides utilisée dans la ceinture cotonnière de Gogounou-Kandi-Banikoara (Adam, 2005). Il s'agit essentiellement de: la cyfluthrine, le lambdacyhalothrine l'indoxacarbe, la spynosine, le profénofos, l'acétamiprid et l'endosulfan.

Par ailleurs, la spectrophotométrie d'absorption atomique a été utilisée selon la méthode de Devez (2004) pour doser le cadmium $(\mathrm{Cd})$, le plomb $(\mathrm{Pb})$, l'arsenic (As), le nickel $(\mathrm{Ni})$ et le zinc $(\mathrm{Zn})$ et le mercure (Hg).

\section{RESULTATS}

Pour le dosage des métaux lourds, les Figures 2 et 3 présentent respectivement les concentrations trouvées dans les sédiments et dans les sols tandis que le Tableau 1 montre leurs teneurs dans l'eau de boisson dans la ceinture cotonnière Gogounou-KandiBanikoara. Pour les pesticides, les Tableaux 2 et 3 montrent respectivement les concentrations trouvées dans les sédiments et les sols.

Les teneurs en métaux lourds obtenues ont été comparées à quelques normes admises regroupées dans le Tableau 4. 


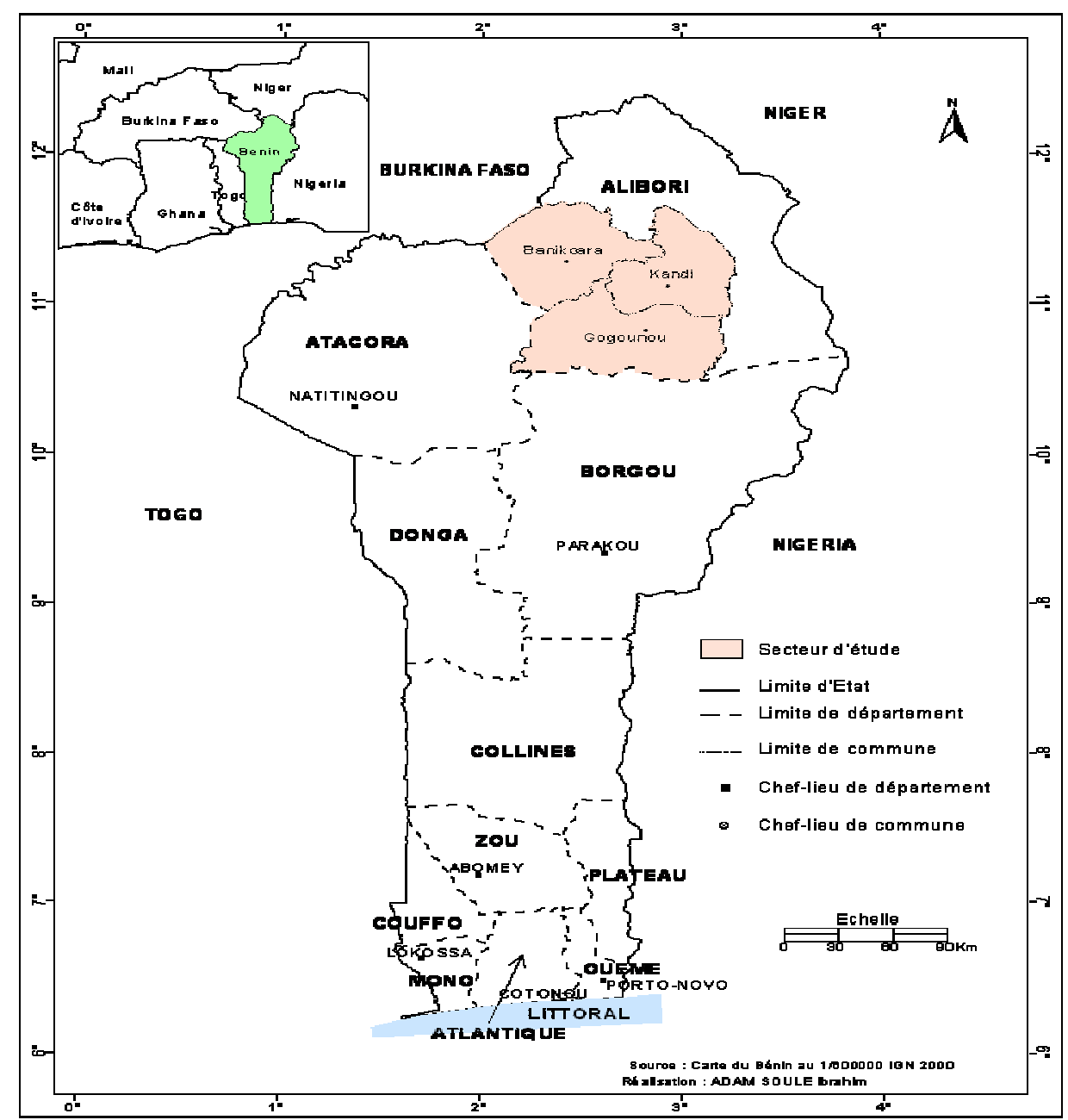

Figure 1: Localisation de la ceinture cotonnière de Gogounou-Kandi-Banikoara.

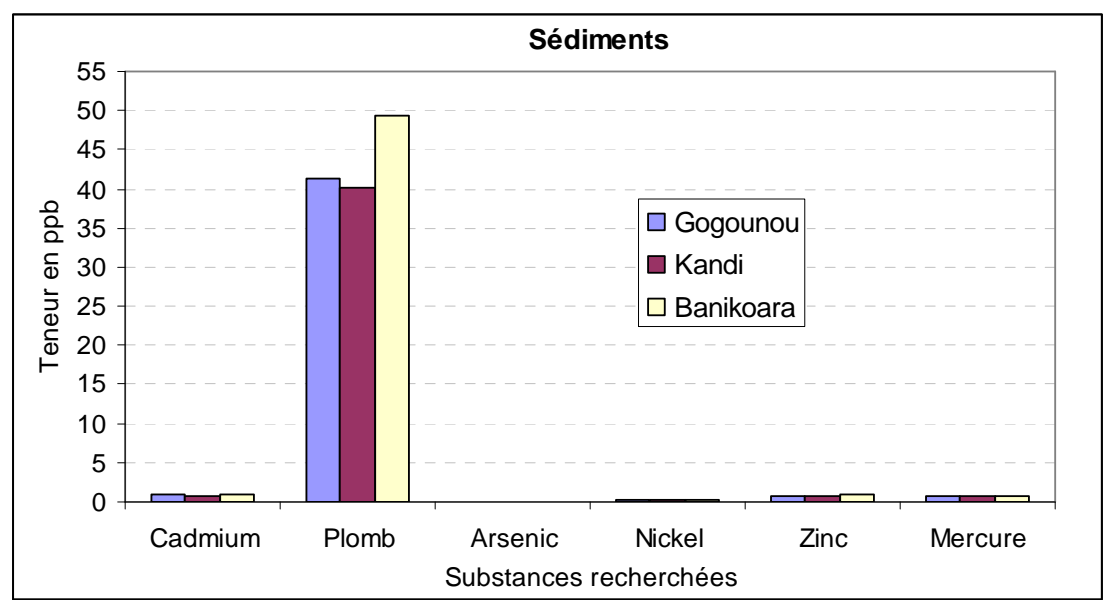

Figure 2 : Concentration des métaux lourds dans les sédiments de la ceinture cotonnière GogounouKandi-Banikoara. 


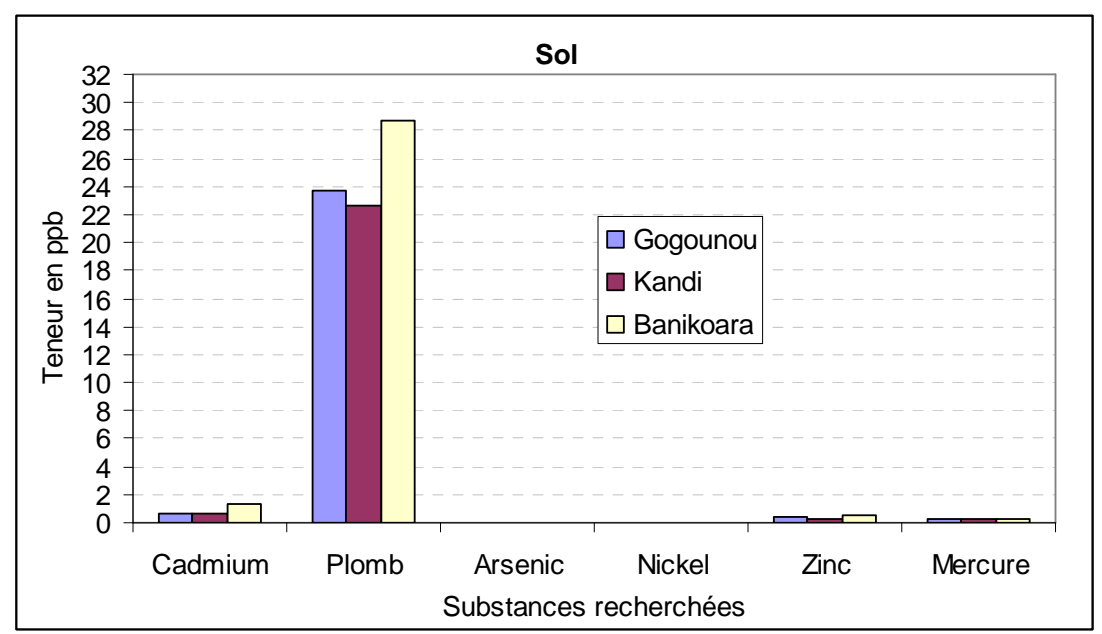

Figure 3 : Concentration des métaux lourds dans les sols de la ceinture cotonnière GogounouKandi-Banikoara.

Tableau 1 : Teneur des métaux lourds dans l'eau de pompe.

\begin{tabular}{llll}
\hline Substances recherchées & Gogounou & Kandi & Banikoara \\
\hline Cadmium $(\mathrm{Cd})$ & $<0,001 \mathrm{ppb}$ & $<0,001 \mathrm{ppb}$ & $<0,001 \mathrm{ppb}$ \\
Plomb $(\mathrm{Pb})$ & $0,12 \mathrm{ppb}$ & $0,13 \mathrm{ppb}$ & $0,13 \mathrm{ppb}$ \\
Arsenic $(\mathrm{As})$ & 00 & 00 & 00 \\
Nickel $(\mathrm{Ni})$ & $<0,002 \mathrm{ppb}$ & $<0,002 \mathrm{ppb}$ & $<0,002 \mathrm{ppb}$ \\
Zinc $(\mathrm{Zn})$ & $<0,1 \mathrm{ppb}$ & $<0,1 \mathrm{ppb}$ & $<0,1 \mathrm{ppb}$ \\
Mercure $(\mathrm{Hg})$ & 00 & 00 & 00 \\
\hline
\end{tabular}

Tableau 2 : Teneur en pesticides dans les sédiments dans la ceinture cotonnière Gogounou-KandiBanikoara.

\begin{tabular}{|c|c|c|c|c|}
\hline \multicolumn{2}{|c|}{ Substances recherchées } & \multirow{2}{*}{$\begin{array}{l}\text { Gogounou } \\
0,195 \mathrm{ppm}\end{array}$} & \multirow{2}{*}{$\begin{array}{l}\text { Kandi } \\
0,197 \mathrm{ppm}\end{array}$} & \multirow{2}{*}{$\begin{array}{l}\text { Banikoara } \\
0,205 \mathrm{ppm}\end{array}$} \\
\hline \multirow{3}{*}{ Pyréthrinoïdes } & Cyfluthrine & & & \\
\hline & Lambdacyhalothrine & $0,036 \mathrm{ppm}$ & $0,036 \mathrm{ppm}$ & $0,040 \mathrm{ppm}$ \\
\hline & Moyenne & 0,1155 & 0,1165 & 0,1225 \\
\hline \multirow{3}{*}{$\begin{array}{l}\text { Insecticides } \\
\text { alternatifs } \\
\text { Pyréthrinoïdes }\end{array}$} & Indoxacarbe & $<0,0010 \mathrm{ppm}$ & $<0,0010 \mathrm{ppm}$ & $<0,0010 \mathrm{ppm}$ \\
\hline & Spynosine & $<0,0010 \mathrm{ppm}$ & $<0,0010 \mathrm{ppm}$ & $<0,0010 \mathrm{ppm}$ \\
\hline & Moyenne & $0,0010 \mathrm{ppm}$ & $0,0010 \mathrm{ppm}$ & $0,0010 \mathrm{ppm}$ \\
\hline \multirow{4}{*}{ Organophosphorés } & Profénofos & $0,093 \mathrm{ppb}$ & $0,093 \mathrm{ppb}$ & $0,096 \mathrm{ppb}$ \\
\hline & Acétamiprid & $0,038 \mathrm{ppm}$ & $0,040 \mathrm{ppm}$ & $0,038 \mathrm{ppm}$ \\
\hline & Organochlorés Endosulfan & $0,12 \mathrm{ppm}$ & $0,12 \mathrm{ppm}$ & $0,15 \mathrm{ppm}$ \\
\hline & Moyenne & $0,079 \mathrm{ppm}$ & $0,08 \mathrm{ppm}$ & 0,094 ppm \\
\hline
\end{tabular}


Tableau 3 : Teneur en pesticides dans le sol dans la ceinture cotonnière Gogounou-KandiBanikoara.

\begin{tabular}{lllll}
\hline Substances recherchées & & Gogounou & Kandi & Banikoara \\
\hline \multirow{3}{*}{ Pyréthrinoïdes } & Cyfluthrine & $0,19 \mathrm{ppm}$ & $0,20 \mathrm{ppm}$ & $0,23 \mathrm{ppm}$ \\
& Lambdacyhalothrine & $0,0023 \mathrm{ppm}$ & $0,0023 \mathrm{ppm}$ & $0,0025 \mathrm{ppm}$ \\
& Moyenne & $0,09615 \mathrm{ppm}$ & $0,10115 \mathrm{ppm}$ & $0,11625 \mathrm{ppm}$ \\
\hline \multirow{2}{*}{$\begin{array}{l}\text { Insecticides alternatifs } \\
\text { aux Pyréthrinoïdes }\end{array}$} & Indoxacarbe & $<0,0010 \mathrm{ppm}$ & $<0,0010 \mathrm{ppm}$ & $<0,0010 \mathrm{ppm}$ \\
& Spynosine & $<0,0010 \mathrm{ppm}$ & $<0,0010 \mathrm{ppm}$ & $<0,0010 \mathrm{ppm}$ \\
& Moyenne & $<0,0010 \mathrm{ppm}$ & $<0,0010 \mathrm{ppm}$ & $<0,0010 \mathrm{ppm}$ \\
\hline \multirow{5}{*}{ Organophosphorés } & Profénofos & $0,52 \mathrm{ppb}$ & $0,49 \mathrm{ppb}$ & $0,51 \mathrm{ppb}$ \\
& Acétamiprid & $0,0010 \mathrm{ppm}$ & $0,0010 \mathrm{ppm}$ & $0,0010 \mathrm{ppm}$ \\
& Organochlorés & $0,08 \mathrm{ppm}$ & $0,08 \mathrm{ppm}$ & $0,08 \mathrm{ppm}$ \\
& Endosulfan & & & \\
& Moyenne & $0,0405 \mathrm{ppm}$ & $0,0405 \mathrm{ppm}$ & $0,0405 \mathrm{ppm}$ \\
\hline
\end{tabular}

Tableau 4 : Normes admises pour quelques métaux lourds.

\begin{tabular}{llllll}
\hline Métaux lourds & Hg & Cd & Pb & Zn & As \\
\hline $\begin{array}{l}\text { Eau potable (en ug/l) } \\
\text { (OMS, 2001) }\end{array}$ & 1 & 3 & 10 & 3000 & 10 \\
\hline $\begin{array}{l}\text { Sédiments en mg/kg (poids sec) } \\
\text { (GESAMP, 1982) }\end{array}$ & $0,05-0,3$ & 0,11 & 19 & 95 & 17 \\
\hline Sol PNEC (Inéris, 2003) & $27-23$ & 12 & 19 & 21 & 15 \\
& $\mu \mathrm{g} / \mathrm{kg}$ & $\mathrm{mg} / \mathrm{kg}$ & $\mathrm{mg} / \mathrm{kg}$ & $\mathrm{mg} / \mathrm{kg}$ & $\mathrm{mg} / \mathrm{kg}$ \\
\hline
\end{tabular}

Le $\mathrm{mg} / \mathrm{l}$ peut être comparé au ppm (mg/kg) tandis que le $\mu \mathrm{g} / \mathrm{kg}$ est l'équivalent du ppb.

\section{DISCUSSION}

De l'analyse des résultats (Figure 2 et Figure 3), il ressort qu'il y a une accumulation principalement du plomb, du cadmium, du mercure et plus faiblement du zinc, de l'arsenic et du nickel dans les sédiments plus que dans les sols. Cela paraît évident si on considère d'une part, que les métaux venus des sols de culture du coton s'accumulent dans les sédiments des eaux adjacentes (Coats, 1991). D'autre part, le plomb est très peu soluble (Ablain, 2002) de même que le mercure (Steinnes, 1995) ; leur accumulation dans les sédiments sera donc relativement plus élevée à cause de leur faible solubilité dans l'eau. Par contre, le cadmium, très soluble dans l'eau (Camobreco et al., 1996) est lessivé et sera donc très faiblement lié aux matières organiques des sédiments et à celles du sol. Cette dissolution du $\mathrm{Cd}$ va augmenter sa concentration dans l'eau de ruissellement et dans les cours d'eau environnants, ce qui constitue un risque pour la population qui utilise ces eaux. En effet, les écosystèmes d'eaux douces sont vulnérables aux métaux, car des composés métalliques insolubles accumulés dans les sédiments peuvent être libérés dans l'eau interstitielle et accroître ainsi la concentration de métaux solubles ou en suspension. Dans tous les cas, cette pollution des écosystèmes aquatiques est dangereuse pour le bétail, la faune aquatique voire tout l'écosystème à cause des phénomènes de bioaccumulation et de 
biomagnification dans les réseaux trophiques (CEDA, 1997). Chez l'homme et le bétail, ces métaux lourds absorbés s'accumulent dans différents organes (les reins, le foie et même le cerveau) et provoquent des troubles divers (Miquel, 2001). Cependant, en comparaison des normes admises (Tableau 4), seules les teneurs en plomb dans les sols et les sédiments dépassent largement les limites. Le mercure, le cadmium et le zinc sont à surveiller. Mais, en dépit de sa faible teneur, le Cd peut migrer sous sa forme soluble de la surface du sol vers les ressources en eaux en profondeur (Ablain, 2002). En effet, l'eau de pompe des trois régions contient du cadmium quoique en faible quantité (Tableau 1). Le plomb y est relativement faible en comparaison à sa concentration dans le sol à cause de sa faible solubilité ; le mercure et l'arsenic y sont heureusement absents et les concentrations du zinc $(0,1 \mathrm{ppb})$ et du nickel $(0,002 \mathrm{ppb})$ restent à surveiller au regard des normes admises (Tableau 4).

Globalement, les concentrations de tous les métaux analysés sont plus importantes dans la commune de Banikoara que dans les communes de Gogounou et de Kandi. Ceci témoigne de l'intensification de l'utilisation des engrais chimiques et des fongicides dans la production agricole à Banikoara où d'ailleurs se trouvent les plus grandes superficies cultivables (Guiwa, 1996).

En ce qui concerne les résultats sur le dosage des pesticides (Tableau 2), il est remarqué une contamination assez régulière mais faible des organophosphorés dans les sédiments prélevés dans les trois communes $(0,1155 ; 0,1165$ et 0,1225 ppm en moyenne pour les pyréthrinoïdes respectivement à Gogounou, Kandi et Banikoara). Il en est de même des insecticides : leur teneur est faible et invariable dans les sédiments des trois régions $(0,0010 \mathrm{ppm})$; c'est le cas aussi des organophosphorés dont les concentrations moyennes sont respectivement 0,$079 ; 0,080$ et 0,094 ppm pour les trois communes.

Dans les sols (Tableau 3), la contamination par les pesticides est aussi régulière. Les teneurs moyennes en pyréthrinoïdes sont 0,$096 ; 0,101$ et 0,116 ppm respectivement pour Gogounou, Kandi et Banikoara. Les teneurs en insecticides n'ont pas varié d'une région à l'autre $(0,0010$ ppm) de même que les concentrations en organophosphorés (0.0405 ppm).

Cette contamination des sédiments et des sols par les pesticides met toutefois en péril la faune du sol, en particulier les décomposeurs, et détériore la qualité des eaux fluviales et souterraines; ce qui représente une menace pour l'écosystème aquatique. De plus, la disparition des vers de terre et des bactéries du sol ralentit la minéralisation, ce qui appauvrit d'avantage le sol et potentialise l'usage intensif des engrais.

La comparaison entre la contamination des sols et des sédiments révèle qu'il y a plus de pesticides dans la deuxième que dans la première composante de l'environnement. Cela paraît évident d'autant plus que c'est seulement en saison pluvieuse que la concentration des pesticides dans le sol est élevée à cause de leur grande utilisation (Tapsoba et Bonzi-Coulibaly, 2006). Les échantillons ont en effet été prélevés pendant la saison sèche. On peut aussi penser comme Coats (1991) que les pesticides s'accumulent dans les sédiments à partir du lessivage des sols.

Par contre, la comparaison du niveau de pollution par les pesticides entre les trois communes montre que les pyréthrinoïdes et les organophosphorés sont plus concentrés dans les sols et les sédiments prélevés dans la commune de Banikoara que dans les autres communes. Cela témoigne une fois encore de l'ampleur de l'utilisation des pesticides dans cette zone (Guiwa, 1996). Mais, ceci peut aussi être la conséquence de la persistance des pesticides en particulier l'endosulfan en saison sèche (Tapsoba et Bonzi-Coulibaly, 2006). D'ailleurs, les pesticides utilisés dans la zone cotonnière Gogounou-Kandi-Banikoara sont constitués essentiellement des matières actives de nature pyréthrinoïdes ou organophoschlorés telles que l'endosulfan de synthèse (Adam, 2005). Des études menées en Afrique montrent également une 
contamination par l'endosulfan à des concentrations de 25 à $457 \mu \mathrm{g} / \mathrm{kg}$ des sols analysés. C'est le cas de la Tanzanie (Mwevura, 2002) et du Burkina (Kishimba, 2005). L'endosulfan a été le seul pesticide organochloré homologué et le plus utilisé en culture cotonnière pendant la campagne agricole 2004-2005 (Adam, 2005), période d'échantillonnage. Sa concentration varie selon les saisons mais plus élevée dans le sol (Savadogo et al. 2006; Van Wyk et al. 2001). Ainsi l'endosulfan, au cours des premières semaines de son application dans un sol pauvre en matière organique, pourrait constituer une menace en cas de pluie pour les ressources en eau et les cultures vivrières adjacentes (Biaou et al. 2003). Il peut constituer ainsi un risque pour la santé (PAN AFRICA, 1999). D'ailleurs, des cas d'intoxications fréquents suivis de décès sont régulièrement enregistrés dans la zone d'étude après chaque campagne (OBEPAB, 2001 ; OBEPAB, 2002).

\section{Conclusion}

Les métaux lourds et les molécules de pesticides sont présents dans les sédiments et les sols dans la ceinture cotonnière de Gogounou-Kandi-Banikoara. Prélevés pendant la saison sèche, les sols ont, certes, faiblement concentré les métaux et les résidus de pesticides à l'exception de l'endosulfan persistant plus à Banikoara qu'à Kandi et à Gogounou. Cette pollution des sols représente en saison humide un risque important de contamination des ressources en eau ainsi que les sédiments des eaux environnantes à cause du lessivage et de l'infiltration. Le sol a en effet des capacités limitées de dégrader les métaux lourds et les pesticides, surtout les organochlorés. Dans ces conditions, l'utilisation intensive des pesticides et engrais chimiques dans la culture du coton constitue un risque pour la santé humaine et animale.

\section{REFERENCES}

Ablain F. 2002. Rôle des activités lombriciennes sur la redistribution des éléments traces métalliques issus de boue de station d'épuration dans un sol agricole. Thèse de doctorat. Univ. de Rennes 1 UMR ECOBIO. Paris. 256p.

Aboudou YMR, Gibiyaye Adam M. 2000. Les stratégies paysannes face à l'avenir des cultures de rente dans le Borgou : Le cas des Sous-préfectures de Bembèrèkè, de Gogounou et Kandi. Mémoire de maîtrise de Géographie, Université Nationale du Bénin, 119 p.

Adam SI. 2005. Impacts environnementaux de la gestion des aires de cultures dans la Commune de Banikoara. Mémoire de DEA, Ecole Doctorale Pluridisciplinaire, Univ. d'Abomey-Calavi, 87p.

Biaou C, Alonso S, Truchot D, Abiola FA, Petit C. 2003. Contamination des cultures vivrières adjacentes et du sol lors d'une pulvérisation d'insecticides sur des champs de coton: cas du triazophos et de l'endosulfan dans le Borgou (Bénin). Revue Méd. Vét., 154(5) : 339-344.

Bourrelier PH, Berthelin J. 1998. Contamination des Sols par les Eléments en Traces : Les Risques et leur Gestion. Académie des Sciences - Techniques et Documentation : Paris ; 440p.

Camobreco VJ, Richards BK, Steenhuis TS, Peverly JH, McBride MB. 1996. Movement of heavy metals through undisturbed and homogenized soil columns. Soil Science, 161: 740-750.

CEDA (Centre pour l'Environnement et Développement en Afrique). 1997. Profil environnemental de la zone côtière ; CEDA, Côte d'Ivoire; p43.

CIPPB. 2008. Etude monographique sur le coton. CIPPB.

Cisse IAA, Tandia ST, Fall ElH, Diop S. 2003. Usage incontrole des pesticides en agriculture periurbaine : cas de la zone des Niayes au Sénégal. Cahiers Agricultures, 12: 181-186.

Coats JR. 1991. Pesticide Degradation Mechanisms and Environmental Activation. In Pesticide Transformation Products. Fate and Significance in the Environment; 11-30. 
Deluisa A, Giandon P, Aichner M, Bortolami P, Bruna L, Lupetti A, Nardelli F, Stringari G. 1996. Copper pollution in italian vineyard soils. Commun. Soil Sci. Plant Anal., 27: 1537-1548.

Devez A. 2004. Caractérisation des risques induits par les activités agricoles sur les écosystèmes aquatiques. Thèse de Doctorat, ENGREF, Montpellier, France, 269p.

DIREN (Direction régionale de l'environnement). 2003. État des lieux des pesticides dans les eaux de la région Rhône-Alpes. Campagne de septembre 2001 à décembre 2002. Eaux et milieux aquatiques. Dossier Diren, Rhône-Alpes, $29 \mathrm{p}$.

Edmond JM, Spivack A, Grant BC, Hu MH, Chen ZX, Chen S, Zhong XS. 1985. Chemical dynamics of the Changjiang estuary. Sediment dynamics of the Changjiang estuary and the adjacent East China Sea. Continental Shelf Science, 4: 17-34.

Ferron P, Deguine JP, Mouté JEM. 2006. Évolution de la protection phytosanitaire du cotonnier: un cas d'école. Cah. Agric., 15(1): 128-134.

Gbesso CA. 2000. Etude comparée de rentabilité socio-économique du coton biologique et du coton conventionnel. $65 \mathrm{p}$.

GESAMP (IMO/FAO/UNESCO/WMO/ WHO/IAEA/UN/UNEP Joint Group of Experts on the Scientific Aspects of Marine Pollution). 1982. The Health of the Oceans. Rep. Stud. (15): 108p. ET UNEP Reg. Seas Rep. Stud, (16) : 108p.

Gnandi K, Tométy-Mensah F, Amey Apoh Y, Edorh P. 2007. Distribution, biodisponibilité et bioaccumulation des métaux lourds dans le système lagunaire de Lomé. J. Rech. Sci. Univ., Lomé (Togo), Série A, 9(1): 67-81.

Guiwa Cl. 1996. Evolution des systèmes de production et mutations socioéconomiques dans la sous-préfecture de Banikoara. Mémoire de maîtrise de
Géographie, Université Nationale du Bénin, 147 p. +2 cartes annexes.

Jager-Mischke I. 1993. Danger de l'emploi des pesticides pour l'homme et la nature. In Pesticides et Agriculture Tropicale, Dangers et alternatives, Dümmler C (ed). PAN \& CTA : Ede (Pays-Bas) \& Hamburg (RFA) ; 23-64.

Kammerbauer J, Moncada J. 1998. Pesticide residue assessment in three selected agricultural production systems in the Choluteca River Basin of Honduras. Environ. Pollut., 103: 171-181.

Kishimba MA. 2005. Environmental pesticide pollution in Tanzania, International workshop on pesticides and other organic pollutants in Africa monitoring and mitigation, Ouagadougou, Burkina Faso.

Miquel G. 2001. Les effets des métaux lourds sur l'environnement et la santé. Rapport d'information $\mathrm{n}^{\circ} 261$ fait au nom de l'Office parlementaire d'évaluation des choix scientifiques et technologiques Sénat (261) : Assemblée Nationale, Paris (France), pp100-261.

Mwevura H, Othman OC, Mhehe GL. 2002. Organochlorine pesticide residues in sediments and biota from the coastal area of Dar es Salaam city, Tanzania. Pollut. Bull., 45: 262-267.

Nebie RC, Yameogo TR, Sie SIB F. 2002. Residus de pesticides dans quelques produits alimentaires de grande consommation au Burkina Faso. Bulletin d'Information de la SOACHIM, 4: 6878.

OBEPAB. 2001. Rapport sur les accidents causés par les pesticides chimiques de synthèse utilisés dans la production cotonnière au Bénin. OBEPAB

OBEPAB. 2002. Le Coton au Bénin: rapport de consultation sur le coton conventionnel et le coton biologique au Bénin. A report for PAN UK's Pesticides Poverty and Livelihoods Project.

OMS/FAO. 2001. Codex Alimentarius Vol 2B Réf. CAC/MRL 3 Rome. 
PAN AFRICA. 1999. Réglementation des pesticides dangereux au Bénin. Monitoring and Briefing $\mathrm{N}^{\circ} 1,24 \mathrm{p}$.

Pazou EYA, Laldyd P, Boko M, Van Gestel CAM, Ahissou H, Akpona S, Van Hattum B, Swart K, Van Straalen NM. 2006. Contamination of fish by organochlorine pesticide residues in the Ouémé River catchment in the Republic of Bénin. Environment International, CODEN ENVIDV, 32(5): 594-599.

PNEC INERIS. 2003. Predicted No-Effect Concentration (PNEC) de l'Institut National de l'Environnement Industriel et des Risques: données toxicologiques et environnementales des substances chimiques. PNEC INERIS.

Saad MAH. 1985. Influence of pollution on Lake Mariout, Egypt III. Heavy metals. Rapp. P. V. Reun. CIESM, 29: 145-147.

Soclo HH, Djibril RB, Issa YM. 2005. International workshop on pesticides and other organic pollutants in Africamonitoring and mitigation, Ouagadougou, Burkina Faso.

Steinnes E. 1995. Mercury. In Heavy Metals in Soils (2e édn), Alloway BJ (éd). John Wiley and Sons, Inc.: New York.

Tapsoba KH, Bonzi-Coulibaly Y. 2006. Production cotonnière et pollution des eaux par les pesticides au Burkina Faso. J. Soc. Ouest-Afr. Chim., 21: 87-93.
Toe AM, Kinane ML, Kone S, Sanfo-Boyarm E. 2004. Le non respect des bonnes pratiques agricoles dans l'utilisation de l'endosulfan comme insecticides en culture cotonnière au Burkina Faso: quelques conséquences pour la santé humaine et l'environnement. Revue Africaine de Santé et de Production Animales, 2: 275-278.

Ton P, Vodouhè SD. 1994. Des opportunités d'établir des liens commerciaux en coton durable entre le Bénin et les Pays Bas. 82 p.

Van der Werf H. 1997. Evaluer l'impact des pesticides sur l'environnement. $C E, \mathbf{3 1}$ : $5-22$.

Van Wyk E, Bouwnman H, Van Der Bank H, Verdoorn GH, Hofmann D. 2001. Persistent organochlorine pesticides detected in blood and tissue samples of vultures from different localities in South Africa. Comparative Biochemistry and Physiology Part C, 129: 243-264.

Vodouhè DS. 1994. Concepts, Atouts et Contraintes de l'Agriculture et du Coton Durable au Bénin. URCF : Cotonou.

Wolska L, Wiergowski M, Galer K, Gorecki T, Namiesnik J. 1999. Sample preparation for GC analysis of selected pesticides in surface water. Chemosphere, 39:1477-1486. 